\title{
TRANSFORMATION GROUPS RESEMBLING THE ADJOINT REPRESENTATION
}

\author{
R. W. SULLIVAN ${ }^{1}$
}

\begin{abstract}
If $G$ is a compact, connected Lie group, the isotropy subgroups of the adjoint representation of $G$ are connected and the dimension of the fixed point set of a maximal torus of $G$ is equal to the the rank of $G$. Results similar to these are given when $G$ acts differentiably on an integral cohomology sphere and has the adjoint representation as weak linear model. This is done by analyzing an induced action of the Weyl group of $G$.
\end{abstract}

1. Let $G$ be a compact, connected Lie group. $G$ acts on itself by conjugation, fixing the identity element and thereby giving rise to an orthogonal representation of $G$ on the tangent space at the identity. This representation, called the adjoint representation, is completely described by its restriction to a maximal torus $T$ of $G$, and this restriction is in turn completely described by the roots of $G$ which are considered as vectors in the Lie algebra of $T$. The Lie algebra of $T$ is contained in the Lie algebra of $G$; since it is fixed by $T$ it is acted on by $N T / T=W$, where $N T$ is the normalizer of $T$ in $G$. The finite group $W$, called the Weyl group of $G$, permutes the roots of $G$ so that if $\alpha$ is a root, so is $\sigma \alpha$ for any $\sigma$ in $W . W$ is generated by the orthogonal reflections through the hyperplanes perpendicular to the (nonzero) roots. If $S_{\alpha}$ is the reflection through the plane perpendicular to $\alpha$ and $\sigma$ is an element of $W, \sigma S_{\alpha} \sigma^{-1}$ is reflection through the root $\sigma \alpha$. Every reflection in $W$ is of the form $S_{\beta}$ for some nonzero root $\beta[2, \mathrm{p} .74]$. $W(G)$ will also be used to denote the Weyl group of $G$.

Let $F(T)$ denote the fixed point set of $T ; G_{x}$ the subgroup of elements of $G$ which fix $x$ (called the isotropy subgroup); $G_{x}^{0}$ the connected component of $G_{x}$ which contains the identity. If $g$ is an element of $G$ which fixes $x$ but does not lie in $G_{x}^{0}$, then $g^{-1} T g=k^{-1} T k$ for some $k$ in $G_{x}^{0}$ since all maximal tori of $G_{x}^{0}$ are conjugate, so $g k^{-1}$ is an element of $W(G)_{x}$ when $x$ is fixed by $T$. If $\alpha$ is a nonzero root and $H_{a}$ is the plane fixed by $S_{a}$, the centralizer in $G$ of the corank one subtorus of $T$ corresponding to $H_{a}$

Received by the editors January 21, 1974.

AMS (MOS) subject classifications (1970). Primary $57 \mathrm{E} 15$.

Key words and phrases. Weyl group, adjoint representation, cohomology sphere.

1 Partially supported by National Science Foundation Grant GU 3171. 
is denoted by $G_{a}$. Since $G_{a}$ is connected [3, pp. 26-31] and $W\left(G_{\alpha}\right)$ is generated by $S_{a}$, any point in $F(T)$ fixed by $S_{a}$ is also fixed by $G_{a}$. Since $W(G)_{x}$ is generated by reflections which fix $x$, we have

Proposition 1. (a) If $x \in F(T)$ is fixed by $S_{a}, G_{a} \subset G_{x}$.

(b) The isotropy subgroups of the adjoint representation are connected.

(c) For $x \in F(T), W(G)_{x}=W\left(G_{x}\right)$.

2. We want to generalize these observations to differentiable actions on cohomology spheres. If $M$ is a differentiable manifold, a diffeomorphism reflection on $M$ is a diffeomorphism $r$ such that (a) $r$ reverses orientation and $r^{2}=1$; (b) the complement of the fixed point set of $r$ is disconnected. We shall refer to diffeomorphism reflections simply as reflections. The fixed point set of a reflection on an integral cohomology sphere $H S^{n}$ is a $Z_{2}$ cohomology sphere by Smith theory. However, a standard argument [6, p. 35] shows that in fact it is an integral cohomology sphere $H S^{n-1}$.

If $W$ is a group which is the Weyl group of a semisimple Lie group $G$, the rank of $W$ is defined as the rank of $G$ (i.e. the dimension of a maximal torus of $G$ ). For such a group $W$ we consider differentiable actions ( $W, \phi$, $\left.H S^{k}\right)$. Such an action is called effective if no element of $W$ fixes every element of $H S^{k}$. Such an action is called normal if (a) each linear reflection in $W$ is a reflection on $H S^{k}$; (b) no product of two distinct linear reflections in $W$ acts trivially. Considering $\phi$ as a homomorphism of $W$ into the group of diffeomorphisms of $H S^{k}$, we say that a reflection on $H S^{k}$ is proper if it is the image under $\phi$ of a linear reflection in $W$.

Theorem 2. If $\left(W, \phi, H S^{k}\right)(k \geq 1)$ is a normal action, then it is effective and each reflection is proper.

Proof. The proof is by induction on the rank of $W$. If rank $W=1$, the theorem follows trivially from the definition. Let rank $W=n>1$ and suppose that the theorem is true for rank $W$ less than $n$. If $S$ is a proper reflection there is a point $x$ in $H S^{k}$ which is fixed by $S$ but which is not fixed by any other proper reflection since the proper reflections have distinct fixed point sets (which meet transversally). If $t$ is any other element of $W$ fixing such an $x$, then $t^{-1} S t=S$. Hence any element of $W$ acting trivially must lie in the center of $W$. If $w$ is such an element, the eigenvalues of $w$ are \pm 1 ; but if $w$ takes +1 as an eigenvalue, then $w$ lies in a Weyl subgroup of $W$ of less rank by Proposition $1(\mathrm{c})$. So we may assume that $w=-I$.

Lemma. The element $-I$ in $W$ can be written as the product of $n$ mutually commuting distinct linear reflections where $n=\operatorname{rank} W$. 
Proof of Lemma. It is sufficient to prove this when $W$ is the Weyl group of a simple Lie group. If $W=W\left(E_{6}\right), W\left(A_{q}\right), q \neq 1$, or $W\left(D_{p}\right), p$ odd, then $-I$ is not an element of $W[2, p .284]$. For the remaining classical groups it is obvious, and since $W\left(B_{4}\right)$ is contained in $W\left(F_{4}\right)$ and $W\left(D_{8}\right)$ is contained in $W\left(E_{8}\right)$, we are left with $G_{2}$ and $E_{7}$. We use the notation of [2, pp. 250-275]. For $G_{2}$ reflect through roots in the set $\left\{\epsilon_{1}, \epsilon_{2}\right\}$. For $E_{7}$ use roots in the set $\left\{\epsilon_{1}-\epsilon_{2}, \epsilon_{1}+\epsilon_{2}, \epsilon_{3}-\epsilon_{4}, \epsilon_{3}+\epsilon_{4}, \epsilon_{5}-\epsilon_{6}, \epsilon_{5}+\epsilon_{6}, \epsilon_{7}-\epsilon_{8}\right\}$.

Retuming to the proof of the theorem, we have $w=R_{1} R_{2} \cdots R_{n}$ acting trivially, $R_{i} R_{j}=R_{j} R_{i}$. Since each $R_{i}$ reverses orientation, $n$ must be even. But each $R_{i}$ acts on the fixed point set of $R_{n}, F\left(R_{n}\right)$, and must reverse its orientation ( for $i \neq n$ ) since it does so locally near a fixed point. Hence the case $n$ even is also impossible.

If $s$ in $W$ is an improper reflection we have essentially the same situation. Since $s^{2}=I$, either $s$ lies in a Weyl subgroup of smaller rank or $s=-I$, so we may write $s=R_{1} R_{2} \cdots R_{n}$ in accordance with the Lemma above. Each $R_{i}$ acts on $F(s)$ and reverses the orientation (or interchanges the two fixed points when $k=1)$. Since the product acts trivially on $F(s)$ we are done.

3. Let $\left(G, \phi, H S^{n}\right)$ be a differentiable action of a compact connected Lie group $G$ on an integral cohomology sphere $H S^{n}$. Let $S(\phi)$ be the cwsystem of $\phi[8] . S(\phi)$ is defined as a collection of linear functionals on the Lie algebra of $T$ which vanish on the Lie algebras of the connected corank one subtori of $T$ which have fixed point sets of greater dimension than that of $T . S(\phi)$ includes the zero functional with multiplicity $d F(T)+1$, where $d F(T)$ denotes the dimension of the fixed point set of $T ; S^{\prime}(\phi)$ denotes the collection of nonzero elements in $S(\phi)$. We assume for the remainder of this section that $S^{\prime}(\phi)=S^{\prime}\left(\operatorname{Ad}_{G}\right)$; the elements of this set then vanish on the hyperplanes perpendicular to the roots of $G$ discussed in $\$ 1$. The fixed point set of $T$ is an integral cohomology sphere acted upon by $W(G)$. The fact that this is a normal action is proved in $[8,1.11]$ and will not be repeated here. It is an easy consequence of a theorem of W.-Y. Hsiang which states that Proposition 1(a) holds for differentiable actions (cf. [5, Proposition 3, p. 349]).

Since $W(G)_{x}$ is generated by the reflections which fix $x$ [4, Theorem 1] and since these are now known to be proper reflections, it follows, just as in the proof of Proposition 1, that the isotropy subgroups are connected and that $W(G)_{x}=W\left(G_{x}\right)$ for $x$ in $F(T)$. That $T$ is a principal isotropy subgroup of $\phi$ now follows from [7, Theorem 3.3]. Since the $W(G)$ action is now 
known to be effective, it follows from Theorem 3 of [4] that $d F(T) \geq \operatorname{rank} G$ - 1. Thus we have

Theorem 3. Let $\left(G, \phi, H S^{n}\right)$ be a differentiable action of a compact connected Lie group with $S^{\prime}(\phi)=S^{\prime}\left(\operatorname{Ad}_{G}\right)$. Then:

(a) $d F(T) \geq \operatorname{rank}(G)-1$;

(b) All isotropy subgroups are connected;

(c) For $x$ in $F(T), W(G)_{x}=W\left(G_{x}\right)$.

(d) $S(\phi)=S\left(\right.$ Ad $_{G}+r$ trivial copies $)$, where $r=d F(T)-\operatorname{rank} G+1=$ $d F(G)+1$.

Remark. Part of the proof of Theorem 3 of [4] has not yet appeared, namely the proof of Theorem $2^{\prime}$. In lieu of this, one can produce a weaker inequality in (a) above as follows: $W(G)$ contains subgroups isomorphic to $Z_{2} \times \cdots \times Z_{2}=\left(Z_{2}\right)^{k}$, where each copy of $Z_{2}$ is generated by a reflection in $W(G)$. If $-I$ is an element of $W(G)$, the Lemma shows that $k=\operatorname{rank} G$; otherwise $k$ is at least $1 / 2 \operatorname{rank} G$. One can apply the formula of Borel [ 1 , Chapter XIII, Theorem 2.3] with $Z_{2}$ coefficients to these subgroups to show that $d F(T)$ is at least $1 / 2 \operatorname{rank} G$.

\section{REFERENCES}

1. A. Borel, et al., Seminar on transformation groups, Ann. of Math. Studies, no. 46, Princeton Univ. Press, Princeton, N. J., 1960. MR 22 \#7129.

2. N. Bourbaki, Éléments de mathématiques. Fasc. XXXIV. Groupes et algèbres de Lie. Chaps. 4, 5, 6, Actualités Sci. Indust., no. 1337, Hermann, Paris, 1968. MR $39 \# 1590$.

3. G. Bredon, Introduction to compact transformation groups, Academic Press, New York, 1972.

4. W.-C. Hsiang and W.-Y. Hsiang, A fixed theorem for finite diffeomorphism groups generated by reflections, The Steenrod Algebra and its Applications (Proc. Conf. to Celebrate N. E. Steenrod's Sixtieth Birthday, Battelle Memorial Inst., Columbus, Ohio, 1970), Lecture Notes in Math., vol. 168, Springer-Verlag, Berlin, 1970, pp. 90-106. MR $42 \# 8519$.

5. W.-Y. Hsiang, On the splitting principle and the geometric weight system of topological transformation groups. I, Proc. Second Conf. on Compact Transformation Groups, part 1, Lecture Notes in Math., vol. 298, Springer-Verlag, Berlin and New York, 1972.

6. N. E. Steenrod, Cohomology operations, Ann. of Math. Studies, no. 50, Princeton Univ. Press, Princeton, N. J., 1962. MR 26 \#3056.

7. R. Sullivan, Differentiable actions of classical groups on spheres, Topology 9 (1970), 155-167. MR 41 \#624.4.

8. Linear models for compact groups acting on spheres, Topology $13(1974), 77-87$.

DEPARTMENT OF MATHEMATICS, STATE UNIVERSITY OF NEW YORK, ALBANY, NEW YORK 12222 